\title{
EFEITO DO EXERCÍCIO FÍSICO INTENSO NAS CONCENTRAÇÕES SÉRICA, SALIVAR E URINÁRIA DE MARCADORES DE LESÃO MUSCULOESQUELÉTICA
}

\author{
Chielle EO, ${ }^{*}$ Maziero JS ${ }^{\dagger}$
}

\section{Resumo}

A lesão muscular esquelética é um evento frequente entre os atletas. O diagnóstico utilizando osmarcadoressanguíneos clássicosàs vezes produzresultadosinsatisfatórios em razão da grande variabilidade interindividual. Portanto, a identificação de biomarcadores confiáveis e agudos é importante. Neste estudo verificou-se a atividade sérica, salivar e urinária de marcadores bioquímicos indicadores de lesão musculoesquelética em resposta ao dano muscular agudo induzido por exercício físico intenso. Realizou-se um estudo transversal com 27 jogadores de futebol americano com idade média de 22,5 anos. Os atletas foram submetidos a um questionário antes da realização da atividade física e em seguida realizouse a avalição de parâmetros clínicos. Antes dos exercícios físicos intensos (T0), 60 minutos (T1) e 24 horas (T2) após o treinamento foram coletadas por punção venosa amostras de sangue que foram acondicionadas em tubos com anticoagulante (heparina) e sem anticoagulante, e posteriormente foi determinada a atividade das enzimas Creatina Quinase (CPK), Adenosina deaminase (ADA), aldolase, Lactato

\footnotetext{
*Doutor e Mestre em Ciências Farmacêuticas Análises Clínicas e Toxicológicas pela Universidade Federal de Santa Maria; Professor na Universidade do Oeste de Santa Catarina; Rua Oiapoc, 211 , 89900-000, São Miguel do Oeste, Santa Catarina, Brasil; eduardochielle@yahoo.com.br † Graduanda em Farmácia pela Universidade do Oeste de Santa Catarina; jorlanamziero@gmail.com
} 
desidrogenase (LDH), Transaminase Glutâmica Oxalacêtica (TGO) e Transaminase Glutâmica Pirúvica (TGP) em amostras de soro, saliva e urina, além da concentração das Substâncias Reativas ao Ácido Tiobarbitúrico (TBARS). Observou-se um aumento significativo da atividade da aldolase, ADA e CPK no soro, da aldolase na saliva e ADA, CPK, TGO e TGP na urina. Também houve um aumento sugnificativo do TBARS salivar e urinário após exercício físico intenso. Em conjunto, os resultados mostraram que a atividade da ADA sérica e urinária, TBARS urinário e salivar, aldolase salivar e TGO e TGP urinário $(p<0.01)$ poderiam ser biomarcadores eficazes e promissores de lesão muscular aguda, permitindo uma avaliação rápida de lesões induzidas pelo exercício físico intenso e fadiga muscular, contribuindo para o monitoramento de atletas e dessa forma, para evitar lesões mais graves e afastamento dos atletas. Palavras-chave: Lesão muscular. Exercício físico. Biomarcadores. Atletas.

\title{
Effect of intensive physical exercise on the serum, salivary and urinary concentrations of lesion markers skeletal muscle
}

\begin{abstract}
Skeletal muscle injury is a frequent event among athletes. The diagnosis using the classic blood markers sometimes produces unsatisfactory results due to the great interindividual variability. Therefore, the identification of reliable and acute biomarkers is important. This study verified the serum, salivary and urinary activity of biochemical markers indicative of musculoskeletal injury in response to acute muscle damage induced by intense physical exercise. A cross-sectional study was carried out with 27 football players with a mean age of 22.5 years. The athletes were submitted to a questionnaire before performing the physical activity and then evaluation of clinical parameters. Before the intense physical training (TO), 60 minutes (TI) and 24 hours (T2) after intense physical training, blood samples were collected by venipuncture that were conditioned in tubes with anticoagulant (heparin) and without anticoagulant and subsequently determined the activity of the enzymes Creatin Kinase (CPK), Adenosine deaminase (ADA), aldolase, Lactate dehydrogenase (LDH), Transaminase Glutamic Oxalacetic (TGO), Transaminase Glutamic Pyruvic (TGP) in serum, saliva and urine samples. Substances Reactive to Thiobarbituric Acid (TBARS). A significant increase in aldolase activity, ADA and CPK in serum, aldolase in saliva and ADA, CPK, TGO and TGP in urine was observed. There was also a significant increase in salivary and urinary TBARS after intense physical exercise. Together, the results showed that serum and urinary ADA activity, urinary and salivary TBARS, aldolase salivary and urinary TGO and TGP $(p<0.01)$ could be effective and promising biomarkers of acute
\end{abstract}


muscle injury, allowing a rapid assessment of physical exercise-induced lesions intense and muscular fatigue, contributing to the monitoring of athletes and thus to avoid more serious injuries and distance of the athletes. Keywords: Muscle injury. Physical exercise. Biomarkers. Athletes.

\section{INTRODUÇÃO}

Todo esporte de alto rendimento tem exigido cada vez mais de seus atletas. As competições esportivas, sejam elas de curta ou longa duração, têm colocado o corpo do atleta sob constante estresse fisiológico, muitas vezes porque essas competições e treinamentos acontecem em um curto espaço de tempo, não permitindo uma regeneração adequada da musculatura.'

Como consequência dos treinamentos e competições em excesso, observase uma sobrecarga muscular, levando a diferentes graus de microtraumas nos músculos, tecido conjuntivo, ossos e articulações. Esse estresse pode progredir de uma fase inicial benigna a uma lesão subclínica, que prejudicará o desempenho do atleta. Uma das prováveis causas para fadiga, dano muscular e inflamação é a ocorrência de rupturas em sarcômeros mais frágeis quando altas forças são aplicadas e com muita frequência. ${ }^{2}$

Com essas rupturas ocorre a liberaçãos de substâncias intracelulares, as quais são sugeridas por muitos autores como parâmetros bioquímicos para monitoração de lesões musculares e que podem ser úteis no acompanhamento bioquímico de lesões musculares..$^{3-5}$ Como expemplo pode ser citada a Creatina quinase (CK), uma vez que treinamentos diários podem resultar em seu aumento sérico. ${ }^{6} \mathrm{Um}$ grande aumento nos níveis séricos de CK combinada com a tolerância diminuída ao exercício poderia ser marcador de overtraining. ${ }^{7}$ Da mesma forma, o exercício físico induz um aumento significativo na Lactado Desidrogenase (LDH). Estudos com atletas de resistência apontam modificações bioquímicas típicas, em 12 a 24 horas após a prática esportiva, caracterizadas por aumento da atividade da LDH. ${ }^{8}$

Dosagens de aldolase e Aspartato AminoTransferase (AST) sanguínea podem ser um indicativo do grau da lesão muscular, e o exercício físico intenso/prolongado pode aumentar temporariamente a taxa plasmática dessas enzimas, permitindo um monitoramento do grau de agressão e tempo de recuperação após esforços físicos intensos. 910

No entanto, o monitoramento bioquímico atual é relativamente não específico e envolve algum desconforto físico e psicológico aos atletas, pois a maioia das análises é realizada em amostras de sangue. Aliado a isso, há uma crescente preocupação com os atletas, especialmente com um diagnóstico precoce e acompanhamento preciso das lesões musculares, sendo que a busca por novos biomarcadores, especialmente em amostras biológicas não ivasivas, e 
que indiquem com precisão e precocidade lesão/fadiga do músculo esquelético, é de grande importância para a área de esportes médicos."

Destaca-se, nesse contexto, a Adenosina deaminase (ADA) como um marcador inflamatório e que embora seja encontrada em pequenas quantidades no tecido muscular, tem sido relacionada ao dano muscular. ${ }^{12}$ A ADA é responsável pela desaminação de adenosina em inosina e é uma enzima quase totalmente citoplasmática, pequenas quantidades estão ancoradas na membrana celular e possui pouca ou nenhuma capacidade de passar pelas membranas, visto que com a ruptura destas, o conteúdo das células extravasa e essa enzima circula na corrente sanguínea em maior quantidade. ${ }^{13}$

Ressalta-se, também, que a prática de exercícios físicos intensos aumenta - consumo de $\mathrm{O}_{2}$ muscular, e isso pode ocasionar lesões oxidativas agudas ou crônicas nas células em razão da produção de espécies reativas de oxigênio (ROS), produzindo um aumento na peroxidação lipídica. ${ }^{14}$ Como biomarcador do estresse oxidativo, mais especificamente da peroxidação lipídica da membrana celular, podem ser avaliadas as substâncias que reagem ao ácido tiobarbitúrico (TBARS); os níveis aumentados desse produto indicam a existência de estresse oxidativo nas células, aumentando o risco de ruptura celular e contribuindo para lesões musculares. ${ }^{15}$

Desse modo, o objetivo com este estudo foi analisar o efeito do exercício físico intenso nas concentrações sérica, salivar e urinária de de marcadores bioquímicos de lesão musculoesquelética em atletas antes e após exercício físico intenso e verificar se essas determinações podem possibilitar uma análise das alterações bioquímicas celulares, permitindo um melhor conhecimento das modificações anatomofisiopatológicas que acontecem nas lesões do aparelho locomotor nos exercícios físicos intensos.

\section{MATERIAL E MÉTODOS}

\subsection{PARTICIPANTES}

Foram incluídos neste estudo 27 atletas do sexo masculino, de uma equipe de futebol americano da Cidade de São Miguel do Oeste, SC. O processo de amostragem foi realizado por conveniência, e todos os atletas assinaram o Termo de Consentimento Livre e Esclarecido. O estudo foi aprovado pelo Comitê de Ética e pesquisa com seres humanos da Universidade do Oeste de Santa Catarina sob o protocolo n. 1.629.054. Para a composição da amostra foram adotados como critérios de inclusão atletas com pelo menos dois anos de treinamento sistematizado, em treinamento de no mínimo quatro vezes por semana e com idades entre 19 e 30 anos. Foram excluídos atletas com histórico recente (mínimo de seis meses) 
de lesões musculares, que passaram por procedimentos cirúrgicos (mínimo de um ano), com processos inflamatórios ou doença de Raynaud e atletas fora da faixa etária mencionada.

\subsection{PROCEDIMENTOS EXPERIMENTAIS}

\subsubsection{Avaliação dos atletas}

Os atletas responderam a um questionário antes da realização das atividades físicas, com os quais foram coletadas informações sobre idade, peso, altura e tempo de atividade física. A pressão arterial dos atletas foi medida antes e depois da atividade física usando um esfigmomanômetro manual e um estetoscópio de marca Premium. A altura dos atletas foi medida em centímetros $(\mathrm{cm})$, em um estadiômetro de parede Professional ES2020 Sanny®, e o peso foi verificado em Quilogramas (kg), na escala G-techC, modelo Glass 180, tipo plataforma.

\subsubsection{Treinamento físico}

Para realizar o treinamento físico, os atletas foram instruíd os a permanecer em um período de descanso de três dias. Inicialmente, os atletas foram submetidos a um aquecimento de dois minutos em bicicleta ergométrica, na sequência foi realizado um treinamento físico intenso. O grupo de atletas foi submetido a uma sequência de exercícios dinâmicos com picos de intensidade sob a supervisão dos pesquisadores e um educador físico. O desempenho dos atletas foi controlado durante todo o treinamento. O treino físico consistiu nos seguintes exercícios: degrau, flexão de braço em bosu, avanço e rosca direta com dumble $(10 \mathrm{~kg})$, desenvolvimento com barras e alteres $(10 \mathrm{~kg})$, tríceps francês com anilha $(15 \mathrm{~kg})$, swing com kettlebell $(12 \mathrm{~kg})$, burpee, agachamento isométrico, arremesso de medicine ball $(5 \mathrm{~kg})$, tríceps banco e corrida em velocidade. Esses exercícios foram escolhidos porque requerem uma grande capacidade muscular para o desenvolvimento. Durante os exercícios, os atletas não descansaram, e a recuperação foi ativa. Foram realizadas várias séries com 20 repetições de cada exercício. Essa sequência de exercícios foi repetida por 60 minutos. A temperatura do ar durante o teste foi de $21^{\circ} \mathrm{C}$, e a umidade relativa foi de $62 \%$.

\subsubsection{Coleta de material biológico}

Amostras de sangue, saliva e urina foram coletadas em três momentos, antes que os exercícios fossem realizados com o atleta descansado (TO), 60 minutos após 
a bateria de exercício físico (T1) e 24 horas após a bateria de exercício físico (T2). Os atletas não estavam em jejum.

\subsubsection{Amostras de sangue}

As amostras de sangue foram coletadas por punção venosa e colocadas em tubos de coleta em tudo seco sem anticoagulante e com gel separador que foi deixado à temperatura ambiente durante 20 minutos e depois centrifugados durante 10 minutos a 4000 rpm para obter o soro.

\subsubsection{Amostras de saliva}

Os indivíduos foram instruídos a enxaguar a boca com água três vezes. Foi fornecido um Salivette ${ }^{\circledR}$, que consiste em um recipiente cônico com filtro absorvente. Os participantes foram instruídos a remover o filtro e colocá-lo na boca por um período de três minutos, conforme recomendado pelo fabricante (Salivette Tubes Sarstedt, Nümbrecht, Alemanha). Posteriormente, o filtro foi transferido para o recipiente e centrífugado. As amostras foram congeladas a $-20^{\circ} \mathrm{C}$ até o momento da análise.

\subsubsection{Amostras de urina}

A urina foi coletada em um frasco estéril (aproximadamente $50 \mathrm{~mL}$ ). Os atletas foram instruídos a descartaro primeiro fluxo de urina e coletar o jato médio diretamente no recipiente fornecido. As amostras foram centrifugadas para remoção de células, bactérias e cristais, e o sobrenadante foi usado para análise laboratorial.

\subsubsection{Análises laboratoriais}

Nas amostras de soro, saliva e urina, foi determinada a atividade de ADA por kit comercial (Ebram Laboratory of Products LTDA® - São Paulo, Brasil), com base na desaminação enzimática de adenosina em inosina por forma cinética em equipamentos BIO2000 (BIOPLUS $\AA$ - São Paulo, Brasil). Os valores foram expressos em U/L. As atividades de CPK, TGO, TGP, LDH foram determinadas por kits comerciais da Labtest Diagnóstica ${ }^{\circledR}$ (Lagoa Santa, MG, Brasil) e a atividade da aldolase foi determinada por kit comercial Aldolase Activity Assay Kit-colorimetric (Abcam discover more - EUA). Todas as determinações foram de forma cinética em equipamento BIO2000 (BIOPLUS - São Paulo, Brasil), e os valores foram expressos em U/L. Os leucócitos e os linfócitos foram analisados e contados por citometria de fluxo de fluorescência no equipamento XS-800i (Sysmex-Roche Diagnostic USA). De acordo com o método estabelecido por autores ${ }^{16}$ de uma pesquisa realizada em 
2001, a peroxidação lipídica foi estimada pela medição das Substâncias Reativas do Ácido Thiobarbitúrico. O produto da reação foi medido espectrofotométricamente a $532 \mathrm{~nm}$, e os resultados foram expressos em nmol/L.

\subsubsection{Análise Estatística}

Os dados são expressos como média \pm desvio padrão ou mediana e intervalo interquartil. O teste de Kolmogorov-Smirnov foi utilizado para examinar a distribuição de variáveis. Comparações de dados de base entre os grupos foram realizadas pela análise de variância de uma via (ANOVA one-way), seguida pelo teste de Tukey (variáveis paramétricas) ou teste de Kruskal-Wallis, seguido pelo teste de Dunn (variáveis não paramétricas). $O$ valor de $p<0,05$ foi considerado estatisticamente significativo. Os dados foram analisados utilizando-se o software Statistica $6.0^{\circledR}$ (StatSoft, Tulsa, EUA).

\section{RESULTADOS}

\subsection{AVALIAÇÃO DOS ATLETAS}

A população do estudo foi composta por 27 voluntários do sexo masculino, com idade média de $22.5 \pm 4.2$, altura média de $1.79 \pm 0.6$, peso médio de $81.9 \pm 13.8$ e tempo de atividade física com média de $4.0 \pm 2.6$ anos. A pressão arterial dos voluntários não apresentou diferença durante os três momentos (TO, T1 e T2) conforme mostra a Tabela 1.

Tabela 1 - Características básicas da população estudada ${ }^{\ddagger}$

\begin{tabular}{lllc}
\hline & T0 & T1 & T2 \\
\hline $\mathbf{N}$ & 27 & & \\
Gênero & Masculino & & \\
Idade (anos) & $22.5 \pm 4.2$ & & \\
Tempo de atividade & $4.0 \pm 2.6$ & & \\
(anos) & & & \\
Peso (kg) & $81.9 \pm 13.8$ & & $123 \pm 13$ \\
Altura (m) & $1.79 \pm 0.6$ & & $76 \pm 9$ \\
PA sistólica (mmHg) & $127 \pm 11$ & $120 \pm 9$ & \\
PA diastólica (mmHg) & $79 \pm 10$ & $76 \pm 8$ & \\
\hline
\end{tabular}

¥ Os dados foram expressos em média e desvio padrão. TO: antes da realização dos exercícios; 11 : 60 minutos após os exercícios físicos; T2: 24 horas após os exercícios físicos; PA: Pressão Arterial. 


\subsection{ANÁLISES LABORATORIAIS}

\subsubsection{Biomarcadores séricos}

As atividades séricas da Aldolase, ADA, CPK aparesentaram-se significativamente aumentados em uma hora e 24 horas após a atividade física intensa. O nível de leucócitos totais e linfócitos aumentou significatimente após uma hora, havendo declíneo significativo após 24 horas. Não foram observadas diferenças significativas na atividade sérica de LDH, TGO, TGP, assim como nas concentrações de TBARS (Tabela 2).

Tabela 2 - Atividade enzimática no soro e quantificação de células sanguíneas ${ }^{\S}$

\begin{tabular}{lrrr}
\hline \multicolumn{1}{c}{ Biomarcador } & \multicolumn{1}{c}{ T0 } & \multicolumn{1}{c}{ T1 } & \multicolumn{1}{c}{ T2 } \\
\hline Aldolase (U/L) & $3.8 \pm 1.0^{\mathrm{ab}}$ & $5.8 \pm 1.6^{\mathrm{b}}$ & $8.1 \pm 4.1$ \\
ADA U/L) & $7.5 \pm 2.0^{\mathrm{ab}}$ & $10.6 \pm 2.6^{\mathrm{b}}$ & $9.0 \pm 3.6$ \\
CPK (U/L) & $167 \pm 71^{\mathrm{a}}$ & $271 \pm 146$ & $259 \pm 166$ \\
LDH (U/L) & $154 \pm 91$ & $125 \pm 68$ & $150 \pm 93$ \\
TBARS (mmol/L) & $309 \pm 118$ & $397 \pm 257$ & $419 \pm 259$ \\
TGO (U/L) & $22.1 \pm 5.3$ & $24.1 \pm 5.5$ & $25.1 \pm 9.0$ \\
TGP (U/L) & $15.4 \pm 7.5$ & $14.1 \pm 8.2$ & $14.1 \pm 8.4$ \\
Leucócitos (mm3) & $8044 \pm 1536^{\mathrm{a}}$ & $9596 \pm 1708^{\mathrm{b}}$ & $7481 \pm 1398$ \\
Linfócitos (mm3) & $2275 \pm 159^{\mathrm{ab}}$ & $3399 \pm 237$ & $2817 \pm 180$ \\
\hline
\end{tabular}

\subsubsection{Biomarcadores salivares}

Na saliva foi observado um aumento significativo na atividade da aldolase, CPK e na concentração de TBARS uma hora após a atividade física, sendo que a CPK e O TBARS persistiram com um aumento ainda maior nas 24 horas após o exercício físico intenso. Não foram observadas diferenças significativas na atividade salivar de LDH, TGO, TGP e ADA (Tabela 3).

\footnotetext{
§ Os dados foram expressos em média e desvio padrão. Os dados foram processados pela análise de variância de uma via (One-Way - ANOVA), seguido do teste de Tukey. TO: antes dos exercícios serem realizados; T1: 60 minutos após exercícios físicos; T2: 24 horas após exercícios físicos.

a $<0.01$ quando comparado com uma hora; ${ }^{b} \mathrm{p}<0.01$ quando comparado com 24 horas.
} 
Tabela 3 - Atividade enzimática salivarll

\begin{tabular}{lrrr}
\hline \multicolumn{1}{c}{ Biomarcador } & \multicolumn{1}{c}{ T0 } & \multicolumn{1}{c}{ T1 } & \multicolumn{1}{c}{ T2 } \\
\hline Aldolase (U/L) & $2,9 \pm 1.3^{\mathrm{a}}$ & $5,4 \pm 2.4^{\mathrm{b}}$ & $3,0 \pm 1.7$ \\
ADA U/L) & $3.8 \pm 0.9$ & $3.9 \pm 0.8$ & $3.7 \pm 0.7$ \\
CPK (U/L) & $4.2 \pm 2.4^{\mathrm{b}}$ & $5.0 \pm 2.7$ & $6.2 \pm 3.0$ \\
LDH (U/L) & $106 \pm 61$ & $133 \pm 69$ & $59 \pm 38$ \\
TBARS (mmol/L) & $19.4 \pm 11.7^{\mathrm{ab}}$ & $26.6 \pm 18.0^{\mathrm{b}}$ & $34.3 \pm 21.6$ \\
TGO (U/L) & $20.3 \pm 9.6$ & $28.2 \pm 7.7$ & $26.6 \pm 8.1$ \\
TGP (U/L) & $5.3 \pm 4.6$ & $6.7 \pm 5.7$ & $5.0 \pm 4.3$ \\
\hline
\end{tabular}

\subsubsection{Biomarcadores urinários}

A atividade urinária da ADA, CPK, TGO, TGP e a concentração de TBARS aumentaram significativamente uma hora após a atividade física intensa. A atividade da ADA e a concentração de TBARS aumentaram 13 e seis vezes, respectivamente, do valor em repouso após uma hora, retornando para níveis basais após 24 horas. Não foram observadas diferenças significativas na atividade urinária de LDH e aldolase (Tabela 4).

Tabela 4 - Atividade enzimática urinária"

\begin{tabular}{lrrr}
\hline Biomarcador & \multicolumn{1}{c}{ T0 } & \multicolumn{1}{c}{ T1 } & \multicolumn{1}{c}{ T2 } \\
\hline Aldolase (U/L) & $2.2 \pm 1.5$ & $1.9 \pm 0.3$ & $1.7 \pm 0.2$ \\
ADA U/L) & $2.8 \pm 1.1^{a}$ & $39.3 \pm 25.2^{\mathrm{b}}$ & $3.2 \pm 2.5$ \\
CPK (U/L) & $2.3 \pm 1.7^{\mathrm{a}}$ & $3.7 \pm 2.4^{\mathrm{b}}$ & $2.1 \pm 2.0$ \\
LDH (U/L) & $8.8 \pm 3.5$ & $6.9 \pm 3.1$ & $6.8 \pm 4.4$ \\
TBARS (mmol/L) & $13.6 \pm 7.3^{\mathrm{ab}}$ & $80.1 \pm 14.3^{\mathrm{b}}$ & $35.5 \pm 10.7$ \\
TGO (U/L) & $6.4 \pm 1.4^{\mathrm{ab}}$ & $18.5 \pm 3.9^{\mathrm{b}}$ & $2.9 \pm 1.2$ \\
TGP (U/L) & $1.6 \pm 0.8^{\mathrm{ab}}$ & $6.9 \pm 2.9^{\mathrm{b}}$ & $0.9 \pm 0.6$ \\
\hline
\end{tabular}

\section{DISCUSSÃO}

Durante os exercícios físicos ocorrem microtraumas teciduais, os quais resultam em uma resposta inflamatória moderada, que tem como finalidade o processo de cicatrização, com consequente adaptação muscular, óssea e do tecido

\footnotetext{
"I Os dados foram expressos em média e desvio padrão. Os dados foram processados pela análise de variância de uma via (One-Way - ANOVA), seguido do teste de Tukey. TO: antes dos exercícios serem realizados; T1: 60 minutos após exercícios físicos; T2: 24 horas após exercícios físicos.

a $<0.01$ quando comparado com uma hora; ${ }^{b} \mathrm{p}<0.01$ quando comparado com 24 horas.

" Os dados foram expressos em média e desvio padrão. Os dados foram processados pela análise de variância de uma via (One-Way - ANOVA), seguido do teste de Tukey. TO: antes dos exercícios serem realizados; T1: 60 minutos após exercícios físicos; T2: 24 horas após exercícios físicos.

a $<0.01$ quando comparado com uma hora; ${ }^{b} \mathrm{p}<0.01$ quando comparado com 24 horas.
} 
conectivo, resultando em processos de recuperação e adaptação fisiológica do atleta, com inúmeros efeitos considerados positivos para a saúde. ${ }^{17}$ No entanto, essa recuperação não é alcançada por indivíduos que se submetem a exercícios físicos intensos e prolongados ou a treinos exaustivos, ou, ainda, que possuem uma frequência de treinamentos muito elevada, ${ }^{18}$ pois comprometem o processo de regeneração tecidual e alterando os processos metabólicos das células, resultando em maior frequência de lesões e diminuição do rendimento do atleta. ${ }^{19}$

$\mathrm{Na}$ ocorrência de uma lesão tecidual ocasionada pelo exercício físico há o rompimento de estruturas contráteis e dos componentes citoesqueléticos da musculatura esquelética, liberando para o sangue proteínas intracelulares. ${ }^{20}$ Uma das proteínas intracelulares liberadas para o sangue é a ADA, uma enzima localizada principalmente no citoplasma celular de praticamente todo o organismo, incluindo a musculatura esquelética; ${ }^{21}$ desse modo, a lesão celular induzida pelo exercício físico propicia o seu extravasamento para o sangue e fluídos biológicos, como descrito aqui pela primeira vez. Neste estudo evidenciou-se que a atividade da ADA sérica e urinária está significativamente aumentada 60 minutos após atividade física intensa, diminuindo significativamente após 24 horas quando comparado com os mesmos indivíduos em descanso.

Três hipóteses podem ser sugeridas para o aumento da ADA no pós-exercício físico. O primeiro ponto a ser destacado é que a ADA é uma enzima inflamatória, envolvida na maturação de células de sistema imunológico, sendo considerada um marcador de inflamação celular. ${ }^{22,23}$ Dessa forma, quando ocorre uma exaustão celular decorrente de um processo inflamatório, há o efluxo de enzimas citosólicas específicas, entre elas a ADA para a corrente circulatória, evidenciando que a atividade da ADA sérica ou em outros fluídos biológicos está diretamente ligada à lesão celular gerada pelo exercício físico. ${ }^{24}$

Um segundo ponto a ser destacado é que no início da ativação do mecanismo inflamatório ocorre a liberação de substâncias vasodilatadoras e pró-inflamatórias, seguidas da ativação dos neutrófilos e posteriormente monócitos e linfócitos que migram para o local da lesão. ${ }^{25}$ Como pôde ser observado na Tabela 2, a contagem de leucócitos totais e os linfócitos aumentaram significativamente após 60 minutos de exercício físico intenso, diminuindo após 24 horas quando comparado com os mesmos voluntários em descanso.

O terceiro fator a ser destacado é que para os músculos, em especial em exercício, a fonte de energia que os mantém funcionando é Adenosina Trifosfato (ATP). Um composto bioquímico que serve para armazenar e utilizar energia. O ATP é necessário para as reações químicas envolvidas em toda contração muscular. ${ }^{14,16}$ Conforme a atividade do músculo aumenta, mais ATP é consumido e precisa ser reposto para que o músculo continue se movimentando, dessa maneira, com uma maior produção de ATP e consequentemente adenosina, há uma aumento da 
atividade da ADA porque o seu substrato está aumentado. Seu aumento faz com que os níveis de adenosina sérica diminuam e consequentemente sua capacidade antioxidante, isso porque a adenosina é um potente antioxidante que vai até as células impedir o estresse oxidativo, porém se seus níveis estiverem diminuídos a célula fica muito mais vulnerável aos danos oxidativos, o que promove perda da fluidez das membranas e liberação de proteínas intracelulares. ${ }^{26}$

A remoção dos produtos de peroxidação lipídica dos tecidos em exercício pode ser, portanto, explicada em termos hemodinâmicos, porque o fluxo sanguíneo para o músculo esquelético aumenta durante o exercício prolongado intenso se comparado ao repouso. Outras vias alternativas de eliminação de lipoperóxidos são o suor e a urina. Contudo, como o exercício físico de longa duração promove desvio do fluxo sanguíneo da região esplênica para a musculatura esquelética, o consumo de TBARS pelo fígado ou sua eliminação pela urina podem ficar dificultados durante o exercício, sendo possivelmente favorecidos após o término do exercício. ${ }^{15}$ Isso explica os altos valores de TBARs detectados no soro de atletas 24 horas após o exercício físico intenso e os valores aumentados na saliva e urina uma hora após o exercício físico.

Outra proteína intracelular liberada para o sangue durante o exercício físico intenso é a CPK, a qual é a principal enzima reguladora do metabolismo anaeróbio, estando localizada no sarcolema e espaço intermembrana da mitocôndria, sendo responsável por catalisar o movimento e transferência de fosfato ao difosfato de adenosina (ADP), formando consequentemente o trifosfato de adenosina (ATP). A liberação de CPK pode ser causada por dano temporário na fibra muscular acompanhado de ruptura na membrana, ou mesmo em razão da morte da fibra muscular. ${ }^{27}$

Aumentos de CPK foram observados em grande número de atletas após treino de força moderado a intenso ou treino aeróbio. ${ }^{5}$ Dependendo do tipo de exercício realizado, o pico de CPK ocorre em momentos diferentes. Após ser liberada, essa enzima chega à corrente sanguínea com uma elevada concentração se comparada às outras proteínas, e seu pico de liberação pode ocorrer entre $24 \mathrm{e}$ 48 horas após o exercício físico. Os resultados obtidos mostraram que em amostras sanguíneas, salivares e urinárias a atividade da CPK se mostrou elevada 60 minutos e 24 horas após o exercício físico (Tabelas 2, 3 e 4).

O exercício físico também pode induzir um aumento significativo na LDH. O grau de aumento depende da intensidade e duração do esforço. ${ }^{28,29}$ Sua atividade muscular está relacionada à composição das fibras musculares, ${ }^{30}$ e sua degradação ocorre principalmente no fígado, especialmente nas células de Kupffer. ${ }^{31,32}$

Neste estudo não se evidenciou aumento da LDH após o exercício físico, no entanto, apesar da presença em grandes quantidades dessa enzima na musculatura esquelética, o aumento da atividade sérica da LDH não é específico para o tecido muscular, porém ela pode ajudar a avaliar a adaptação ao treinamento..$^{11,33}$ 
Considera-se importante a taxa plasmática da aldolase para monitorar lesões muscular, visto que essa enzima pode aumentar temporariamente após o exercício físico intenso/prolongado, e verificou-se que após uma corrida de $50 \mathrm{~km}$, sujeitos treinados atingiam pico máximo de concentração sérica de aldolase 24 horas depois; os sujeitos não treinados, ou menos treinados, só atingiam esse pico em 48 horas após a corrida. Porém, sujeitos treinados apresentavam logo após o esforço níveis significativamente superiores aos basais, enquanto para indivíduos não treinados os valores após o esforço eram idênticos aos valores de partida. Isso significa que o tempo de extravasamento celular dessas enzimas para $\circ$ sangue não é idêntico em todos os sujeitos. ${ }^{34}$ De maneira geral, essa enzima tem sido usada em conjunto com a CPK para avaliar o status da adaptação muscular ao treinamento." Foi possível observar neste estudo que a atividade sérica e salivar aumentou significativamente 60 minutos após atividade física intensa, e a nível sérico a atividade dessa enzima permaneceu aumentada após 24 horas do término do exercício, enquanto na saliva a atividade dessa enzima diminuiu significativamente após 24 horas (Tabelas 2,3 e 4).

A TGO e a TGP também podem dar indicação de dano muscular, sendo - aumento relacionado à maior permeabilidade do tecido muscular após o exercício físico intenso. Elas são as enzimas que catalisam a conversão da porção nitrogenada de um aminoácido para um resíduo de aminoácido. São liberadas de músculos lesionados e, portanto são utilizadas como marcadores de dano muscular após exercício. ${ }^{35}$ Nos dados aqui apresentados é possível observar o aumento de TGO e TGP 60 minutos após o exercício (Tabela 4) e a diminuição 24 horas após o exercício físico em amostras salivares e urinárias.

\section{CONCLUSÃO}

Neste estudo avaliou-se a concentração de TBARS e a atividade de ADA, CPK, aldolase, LDL, TGO e TGP sérica, urinária e salivar, e verificou-se que essas determinações podem permitir uma análise das alterações bioquímicas celulares, auxiliando no conhecimento das mudanças anatomopatológicas que ocorrem em lesões do aparelho locomotor nos intensos exercícios físicos.

Com base nisso, esses biomarcadores podem ser dosados em amostras não invasivas e em momentos diferentes, pois, como os resultados reportaram, alguns biomarcadores aumentam 60 minutos após o exercício físico intenso, contribuindo, dessa forma, para um diagnóstico precoce e o monitoramento de possíveis lesões musculares, evitando exaustões musculares mais graves em atletas e melhorando seu desempenho. Profissionais envolvidos com os atletas, como médicos e treinadores físicos, serão capazes de organizar uma rotina de treinamento que permita ao atleta uma melhor adaptação metabólica para satisfazer a demanda acelerada 
de energia, bem como a remoção de metabólitos desnecessários para o corpo, sem causar tanto dano ao corpo do atleta. Dessa forma, os resultados são positivos e promissores, uma vez que o monitoramento bioquímico atual é relativamente não específico e envolve algum desconforto físico e psicológico.

Ressalta-se que os resultados em amostras de urina e saliva são tão precisos quanto em amostras de sangue, porém não geram ao atleta nenhum desconforto físico e podem ser realizados diariamente ou semanalmente, o que permite um melhor conhecimento das modificações anatomofisicopatológicasqueacontecem nas lesões do aparelho locomotor nos exercícios físicos intensos.

\section{Agradecimentos}

Os autores agradecem à Universidade do Oeste de Santa Catarina (Unoesc), por seu apoio neste estudo. Além disso, a todos os voluntários que participaram deste estudo, em especial ao Grupos de Atletas Indians, de São Miguel do Oeste, SC.

\section{REFERÊNCIAS}

1.Wilcock IM, Cronin JB, Hing W. A. Physiological Response to Water Immersion: A Method for Sport Recovery. Sports Med. 2006; (369):747-65.

2. Lazarim FL, et al. The upper values of plasma creatine kinase of professional soccer players during the Brazilian National Championship. J Sci Med Sport. 2009; (12):85-90.

3. Peake J, Nosaka K, Suzuki K. Characterization of inflammatory responses to eccentric exercise in humans. Exerc Immunol Rev. 2005, 11:63-85.

4. Petibois $C$, Cazoria $G$, Deleris $G$. The biological and metabolic adaptations to 12 months training in elite rowers. Int J Sports Med. 2003; 24:36-42.

5. Hartmann U, Mester, J. Training and overtraining markers in selected sport events. Med Sci Sports Exerc. 2000; 32(1):209-15. 
6. Kratz A, et al. Effect of marathon running on hematologic and biochemical laboratory parameters, including cardiac markers. Am J Clin Pathol. 2002; 118:856-63.

7. Brancaccio P, Maffulli N, Limongelli FM. CK monitoring in sport medicine. Br Med Bull. 2007; 81-82(1):209-30.

8. Hurley BF, Redmond RA, Pratley RE, Treuth MS, Rogers MA, Goldberg AP. Effects of strength training on muscle hypertrophy and muscle cell disruption in older men. International J Sports Med. 1995; 16(6):378-84.

9. Plotnikov EY, Chupyrkina AA, Pevzner IB, Isaev NK, Zorov DB. Myoglobin causes oxidative stress, increase of NO production and dysfunction of kidney's mitochondria. Biochim Biophys Acta. 2009; 1792(8):796-803.

10. Rosa NG, Silva G, Teixeira A, Rodrigues F, Araújo JA. Rabdomiólise. Acta Med Port. 2005; 18:271-82.

11. Rodrigues dos Santos JA. Avaliação do Processo de recuperação de alguns indicadores hematológicos três dias após a conclusão de uma ultramaratona de 100km. Rev Port Med Desp. 2001; 19:83-94.

12. Pizza $F$, et al. Muscle inflammatory cells after passive stretches, isometric contractions, and lengthening contractions. J Appl Physiol. 2002; 92:1873-8.

13. Baroni BM, Leal ECP, Jr, Generosi RA. Grosselli G, Censi S, Bertolla F. Efeito da crioterapia de imersão sobre a remoção do lactato sanguíneo após exercício. Rev Bras Cineantropom Desempenho Hum. 2010; 12:179-85.

14. He F, Li J, Liu Z, Chuang CC, Yang W, Zuo L. Redox Mechanism of Reactive Oxygen Species in Exercise. Rev Front Physiol. 2016 Nov 7; 7:486. 
15. Alleman RJ, Katunga LA, Nelson MA, Brown DA, Anderson EJ. The "Goldilocks Zone" from a redox perspective-Adaptive vs. deleterious responses to oxidative stress in striated muscle. Rev Front Physiol. 2014 Sep 18; 5:358.

16. Lapenna GD, Ciofani SD, Pierdomenico MA, Giamberardino MA, Cuccurullo F. Reaction conditions affecting the relationship between thiobarbituric acid reactivity and lipid peroxides in human plasma. Free Radic Biol Med. 2001; $31: 331-5$.

17. Nóbrega ACL. The sub-acuteeffects os exercise: concept, characteristics, and clinical implications. Exerc Sport Sci Rev. 2005;33:84-7.

18. Rogero MM, Mendes RR, Tirapegui J. Aspectos neuroendócrinos e nutricionais em atletas com overtraining. Arq Bras Endocrinol Metabol. 2005; 49:359-68.

19. Tidball JG. Inflamatory processes in muscle injury and repair. Am J Physiol Regul Integr Comp Physiol. 2005; 288:R345-53.

20. Rodrigues dos Santos JA. Alterações agudas induzidas por uma corrida de 50 km em alguns parâmetros hematológicos, bioquímicos e urinários em sujeitos com diferentes nivies de treino. Rev. Port. Med Desp. 2004; 22:1-22.

21. Hartmann A, et al. Recommendations for conducting the in vivo alkaline Comet assay.Mutagenesis. 2003; 18:45-51.

22. Chielle EO, Bonfanti G, Bona KS, Moresco RN, Moretto MB. Adenosine deaminase, dipeptidyl peptidase-IV activities and lipid peroxidation are increased in the saliva of obese young adult. Clin Chem Lab Med. 2015 Jun; 53(7):1041-7. 
23. Ferreira CK, et al. Influence of short duration acute exercise on the number, viability, functionality and apoptosis of neutrophils in sedentary rats. J Exerc Physiol. 2007; 10:27-36.

24. Stupka N, Tarnopolsky MA, Yardley NJ, Phillips SM. Cellular adaptation to repeated eccentric exercise-induced muscle damage. J. appl. physiol. 2001; 91:1669-78.

25. Kuno M, Seki N, Tsujimoto S, Nakanishi I, Kinoshita T, Nakamura K. Antiinflammatory activity of non-nucleoside adenosine deaminase inhibitor FR234938. Eur J Pharmacol. 2006; 534:241-9.

26. Alleman RJ, Katunga LA, Nelson MA, Brown DA, Anderson EJ. The "Goldilocks Zone" from a redox perspective-Adaptive vs. deleterious responses to oxidative stress in striated muscle. Rev Front Physiol. 2014 Sep 18; 5:358.

27. Mcneil PL, Khakee R. Disruptions of muscle fiber plasma membranes. Role in exercise - induced damage. Am J Pathol. 1992; 140(5):1097-109.

28. Priest JB, Oei TO, Moorehead WR. Exercise-induced changes in common laboratory tests. Am. J. Clin. Pathol. 1982; 77(3):285-9.

29. Stokke, O. Clinical chemical changes in physical activity. Scand J Public Health. 1982; 29:93-101.

30. Costill DL, Daniels J, Evans W, Fink W, Krahenbuhl G, Saltin, B. Skeletal muscle enzymes and fiber composition in male and female track athletes. J Appl Physiol. 1976; 40(2):149-54.

31. Smit MJ, Beekhuis H, Duursma AM, Bouma JM, Gruber, M. Catabolism of circulating enzymes: plasma clearance, endocytosis, and breakdown of lactate dehydrogenase-1 in rabbits. Clin Chem. 1988; 34:2475-80. 
32. Smit MJ, Duursma AM, Bouma JM, Gruber M. Receptor-mediated endocytosis of lactate dehydrogenase M4 by liver macrophages: a mechanism for elimination of enzymes from plasma. Evidence for competition by creatine kinase MM, adenylate kinase, malate, and alcohol dehydrogenase. J Biol Chem. 1987; 262:13020-6.

33. Silva IAC, Dias RVC, Soto-Blanco B. Determinação das atividades séricas de creatina quinase, lactato desidrogenase easpartato aminotransferase em equinos de diferentes categorias de atividade. Arq Bras Med Vet Zootec. 2007; $59(1): 250-2$.

34. Leppanen EA. Experimental basis of standardized specimen collection: the effect of short moderate exercise on serum K, Na, ASAT, ALAT, CK and LD. Scand J Clin Lab Invest. 1989; 49(3):287-92.

Data de submissão: 16 de março de 2018 Avaliado em: 02 de maio de 2018 (AVALIADOR A) Avaliado em: 03 de maio de 2018 (AVALIADOR C) Aceito em: 07 de maio de 2018 